\title{
Fine structure of the gamonts of Eimeria (s. 1.) vanasi, a coccidium from the intestine of cichlid fishes
}

\author{
Ilan Paperna \\ Department of Animal Sciences, Faculty of Agriculture of the Hebrew University of Jerusalem, Rehovot, 76-100, Israel
}

\begin{abstract}
The fine structure of gamont-forming merozoites, macrogamonts and microgamonts of Eimeria (s. 1.) vanasi, a coccidium found in the intestines of hatchery-raised fry of Oreochromis aurea $x$ nilotica, is described. The apical ends of parasitophorous vacuoles containing young macrogamonts extend, together with the apposed host cell boundary, through the brush border above the epithelial layer The macrogamont cytoplasm contains organelles characteristic of coccidian macrogamonts, including organelles which are structurally identical to Type 1 and 2 wall-forming bodies. Ribosomelined ducts and Type 1 wall-forming-like organelles appear to be involved in the formation of the envelope around the macrogamonts. Microgamont fine structure conforms with that of coccidian microgamonts found in other vertebrates.
\end{abstract}

\section{INTRODUCTION}

Eimeria (s. l.) vanasi Landsberg \& Paperna, 1987 parasitizes the intestine of juveniles of African cichlid fishes. Earlier communications report light microscopy (LM) studies of the endogenous developmental stages and of the oocyst (Landsberg \& Paperna 1987), as well as an transmission electron microscopic (TEM) observation revealing numerous tubules in the host cell cytoplasm connected to funnels located at the rim of the parasitophorous vacuole (PV) (Paperna \& Landsberg 1987). The fine structure of meronts and merozoites is described by the same author in a concurrent communication. In the present communication the fine structure of macrogamonts and microgamonts is reported and discussed.

\section{MATERIALS AND METHODS}

Infected fish, fry of Oreochromis aurea $\times$ nilotica (the cultured 'tilapia') were obtained from hatcheries in Beit Shaan valley (Landsberg \& Paperna 1987). For TEM, pieces from the anterior gut were fixed in Karnowski for $24 \mathrm{~h}$ at $4 \mathrm{C}$, washed repeatedly in $0.1 \mathrm{M}$ cacodylate buffer at pH 7.4, and post-fixed in $1 \%$ osmium tetroxide in the same buffer, for $1 \mathrm{~h}$. After rinsing in buffer, the material was dehydrated in ethanol and embedded in Epon. Thin sections, cut by an LKB III ultratome with diamond knife, were stained on a grid with uranyl acetate and lead citrate and examined with a Jeol 100CX TEM.

\section{RESULTS}

\section{Merozoites}

Merozoites developing into gamonts, 4.5 to $5 \times 2$ to 3 $\mu \mathrm{m}$ in size (Fig. 1), are bounded by 2 unit membranes. They have a large vesicular nucleus with homogeneously distributed chromatin and a distinct nucleolus, rhoptries exhibiting heterogeneous electron density, and faintly osmiophilic micronemes. The cytoplasm also contains a dense network of rough endoplasmic reticulum (ER), peripherally distributed mitochondria and a variable amount of lipid vacuoles. The wall of the PV consists of a pronounced membrane interrupted by a few tubular system funnels.

\section{Young macrogamonts}

PV containing the young macrogamonts ( 4 to $8 \times 3$ to $6 \mu \mathrm{m}$ ) occur beneath the brush border surface of the 

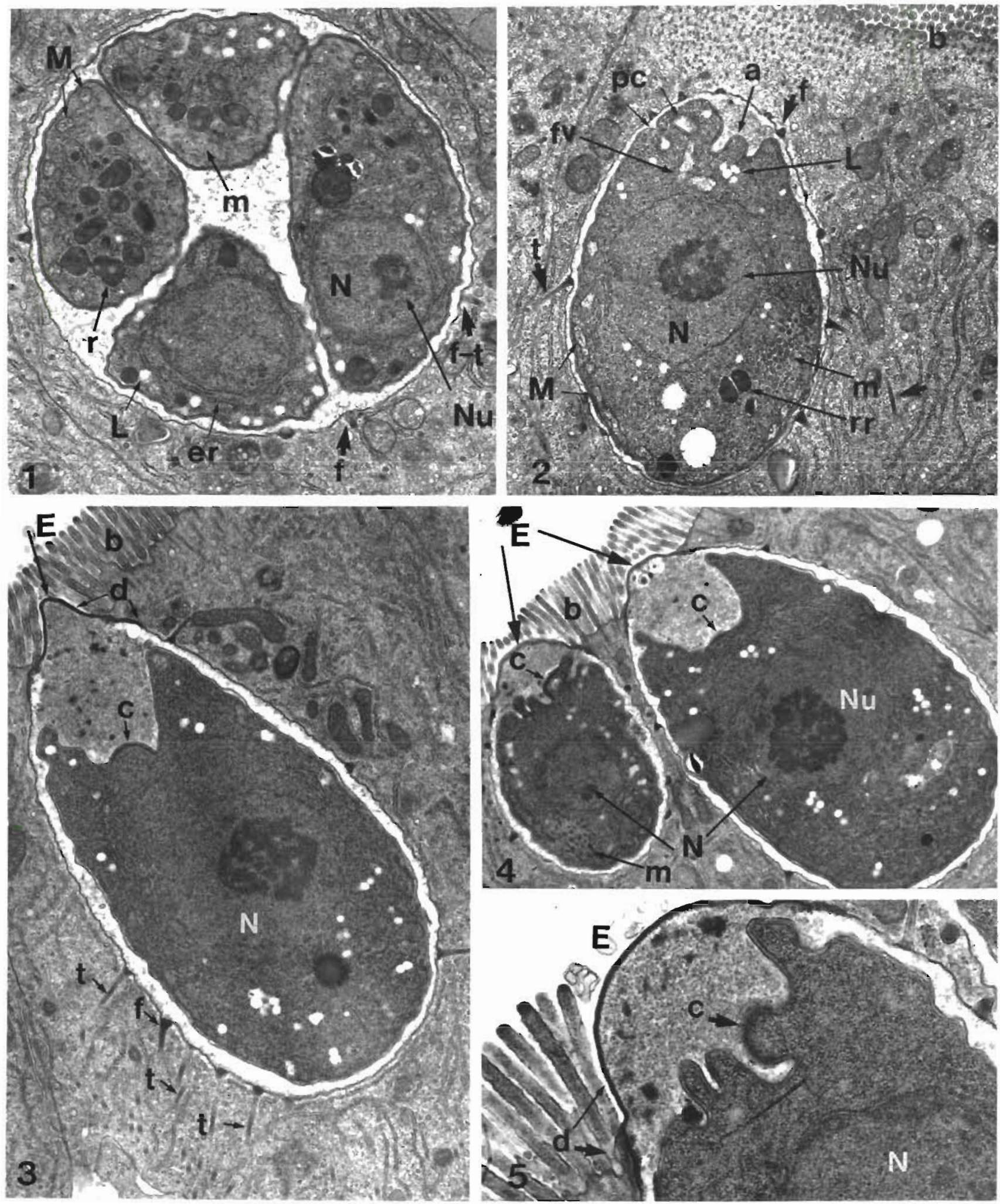

Figs 1 to 5 Exmena (s. l.) vanası. Fig. 1. Progeny of merozoltes which will later develop into gamonts, within a PV (parasitophorous vacuole) connected to funnels ( $f-t)$ of the tubular system $(\times 13900)$ Fig 2 Young macrogamont located beneath the brush border zone $(\times 11300)$. Figs. 3 and 4 . Young macrogamonts within a PV extending into the brush border zone (E); PV boundary is connected to an extensive tubular system ( $\times 8300$ and 11200 , respectively). Fig. 5. Enlargement of the apical end of the PV extending into the brush border zone $(\times 25500)$ Symbols are $(a)$ folded anterior end, (b) brush borderi (c) anterior plaque; (d) thickened wall; (er) endoplasmic retıculum; (f) parasitophorous funnels; (fv) food vacuole; (L) lipid vacuole; (M) mitochondria; (m) micronemes, (N) nucleus; (NU) nucleolus; ( $r$ ) rhoptnes, (rr) resıdual rhoptries; (t) tubular system 
epithelial cells (Figs. 2 to 4 ). The PV is bounded by a unit membrane interspersed with funnels connected to an elaborate tubular system (Figs. 2 and 3 ). The apical end of some of the gamont-containing PVs rises above the epithelial host cell brush border surface along with the apposed epithelial cell boundary membrane (Figs. 3 and 4). This surfacing portion is void of microvilli, and the interior side of its wall is thickened by an overlay of osmiophilic substance, to some distance below the level of the microvillar boundary of the host cell (Figs. 3 and 5). The anterior end of the parasitophorous vacuole contains granular debris (Figs, 3 and 5). The young macrogamont is bounded by 2 unit membranes, its anterior end is depressed (Figs. 3 and 4 ) or folded (Figs. 2 and 5), and a thick plaque is formed at the anterior tip, on the inner boundary membrane (Fig. 3). Some folds form pinocytotic-like depressions (Fig. 2). The large nucleus contains a central, fragmented nucleolus. The cytoplasm contains a network of rough ER, a few food vacuoles at the anterior end (Fig. 2), peripherally distributed mitochondria (Fig. 2), aggregates of faintly osmiophilic micronemes (Figs. 2 and 4), a number of small lipid vacuoles, and a few dense bodies (Figs. 2 and 3), presumably residues of rhoptries.

\section{Macrogamonts}

Macrogamonts ( 8 to $14 \times 7$ to $10 \mu \mathrm{m}$ ) (Figs. 6 to 15 ) are bounded by a thin, single-layered wall and enclosed in a bilaminated envelope (Figs. 12 to 15 ) which is attached to points on the macrogamont surface at apparently regular intervals (Figs. 6 and 7). The space between the macrogamont outer envelope and the wall of the PV contains a flocculent substance (Figs. 8 and 15). The wall of the PV, which consists of a single membrane, is interspersed with funnels from the tubular system (Fig. 15). The nucleus is usually centrally located (Fig. 7) and contains a large nucleolus (Fig. 9). Adnuclear bodies occur at the periphery of the nucleus and seem to be enclosed in extensions of the nucleolemma (Figs. 7 and 8). Canaliculi form a few aggregates at the periphery of the nucleus (Figs, 7 and 11). Mitochondria occur immediately beneath the cell wall (Figs. 6 and 15). Ducts or canals lined with ribosomes (Figs. 6 and 8), together with a small vesicle containing electron-dense material (Figs. 12 to 14), open to the point of attachment between the outer envelope and the cell wall (Fig. 8). Similar small vesicles of electron-dense material occur in the internal cytoplasm (Figs. 8 and 9). These vesicles resemble Type 1 wall-forming bodies (WF1) of higher vertebrate coccidia. Larger round bodies with an electron-dense core, located within the rough ER cisternae (Figs. 11, 12 and 14), are structurally identical to the Type 2 wall-forming bodies (WF2)

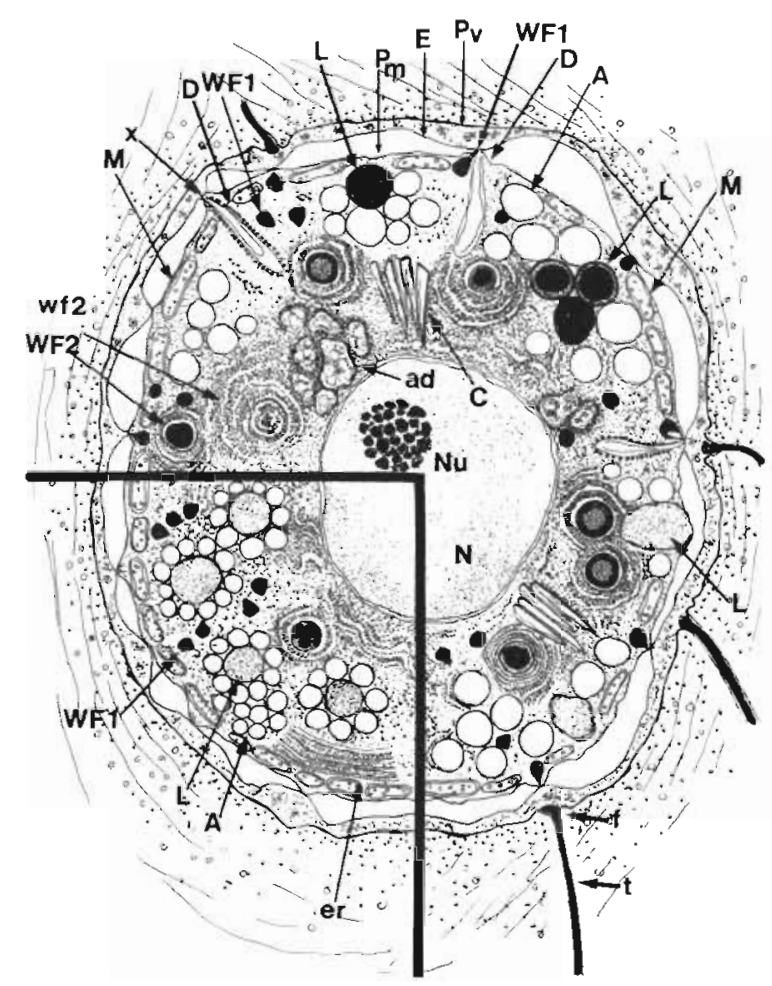

Fig. 6. Eimeria (s. 1.) vanasi. Outline of mature macrogamont organization showing (A) amylopectin granules; (ad) adnuclear bodies; (C) canaliculi; (D) ribosome-lined ducts; (E) envelope; (er) endoplasmic reticulum; (f) funnels; (L) lipid vacuoles; $(M)$ mitochondria; $\left(p_{\mathrm{m}}\right)$ macrogamont's cell boundary; $\left(p_{v}\right)$ wall of parasitophorous vacuole; (t) tubular system; (WF1) Type 1 wall-forming-like bodies; (WF2) Type 2 wallforming-like bodies; (wf2) aggregating WF2-like body; $(x)$ attachment points of envelope

of higher vertebrate coccidia. An aggregation of these bodies is seen in some of the large cisternae (Fig. 10). The rough ER forms a dense network of parallel branches in the peripheral cytoplasm (Figs. 5, 6 and 9) and a winding network of ducts and cisternae in the perinuclear zone (Figs. 7 and 8 ). In some macrogamonts small amylopectin granules surround large, partly-extracted lipid vacuoles (Fig. 9), while in others the granules are larger and are distributed independent of highly osmiophilic lipid vacuoles (Figs. 7 and 8).

\section{Microgamonts}

Microgamonts were only found at an early stage of microgametogenesis. The microgamonts (10 to $11 \times 8$ to $9 \mu \mathrm{m}$ ) (Figs. 16 to 19 ), are bounded by a single membrane. The walls of PVs containing microgamonts are fringed with funnels from the tubular system (Fig. 16). Nuclei with peripherally concentrated heterochromatin aggregate beneath the surface of the micro- 


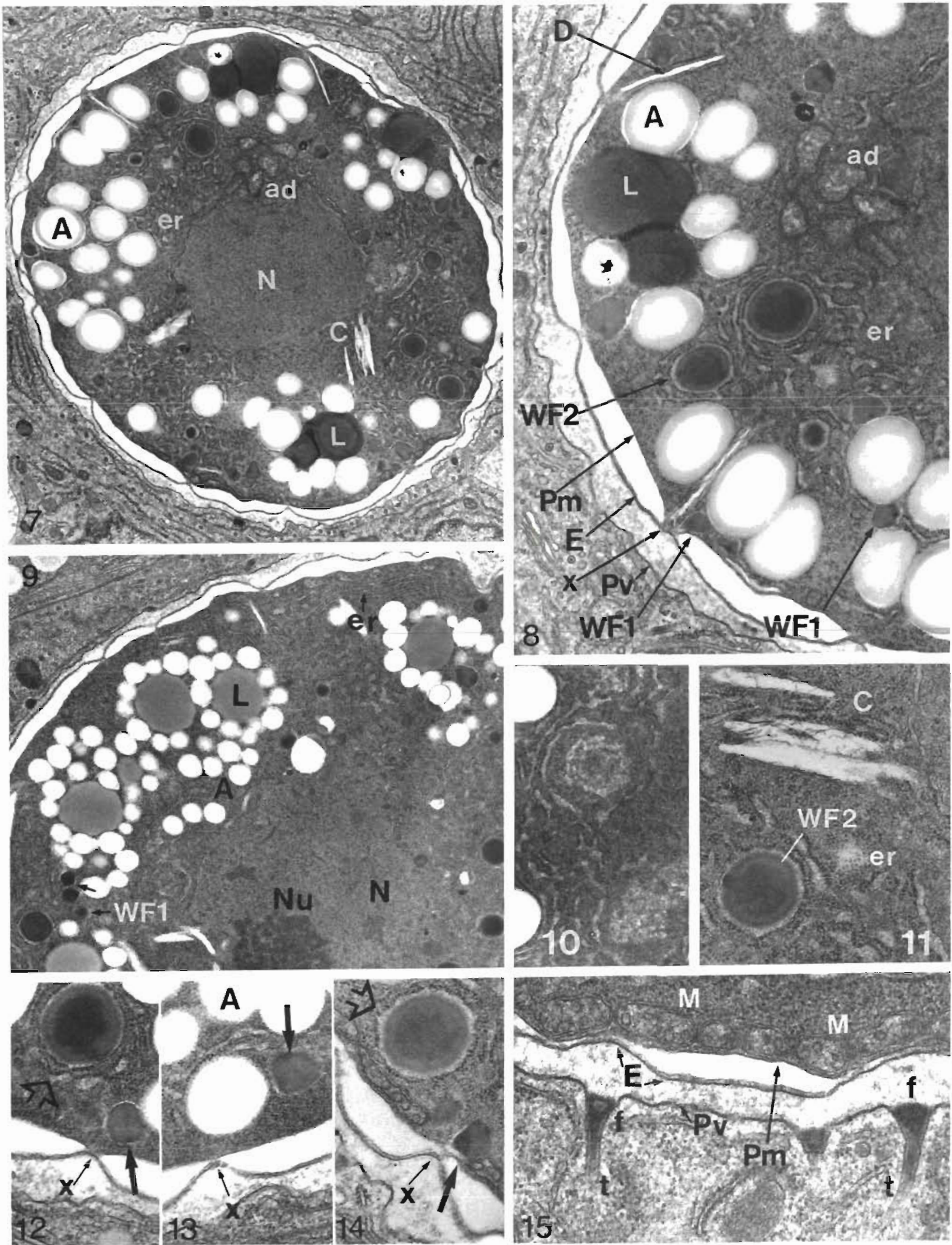



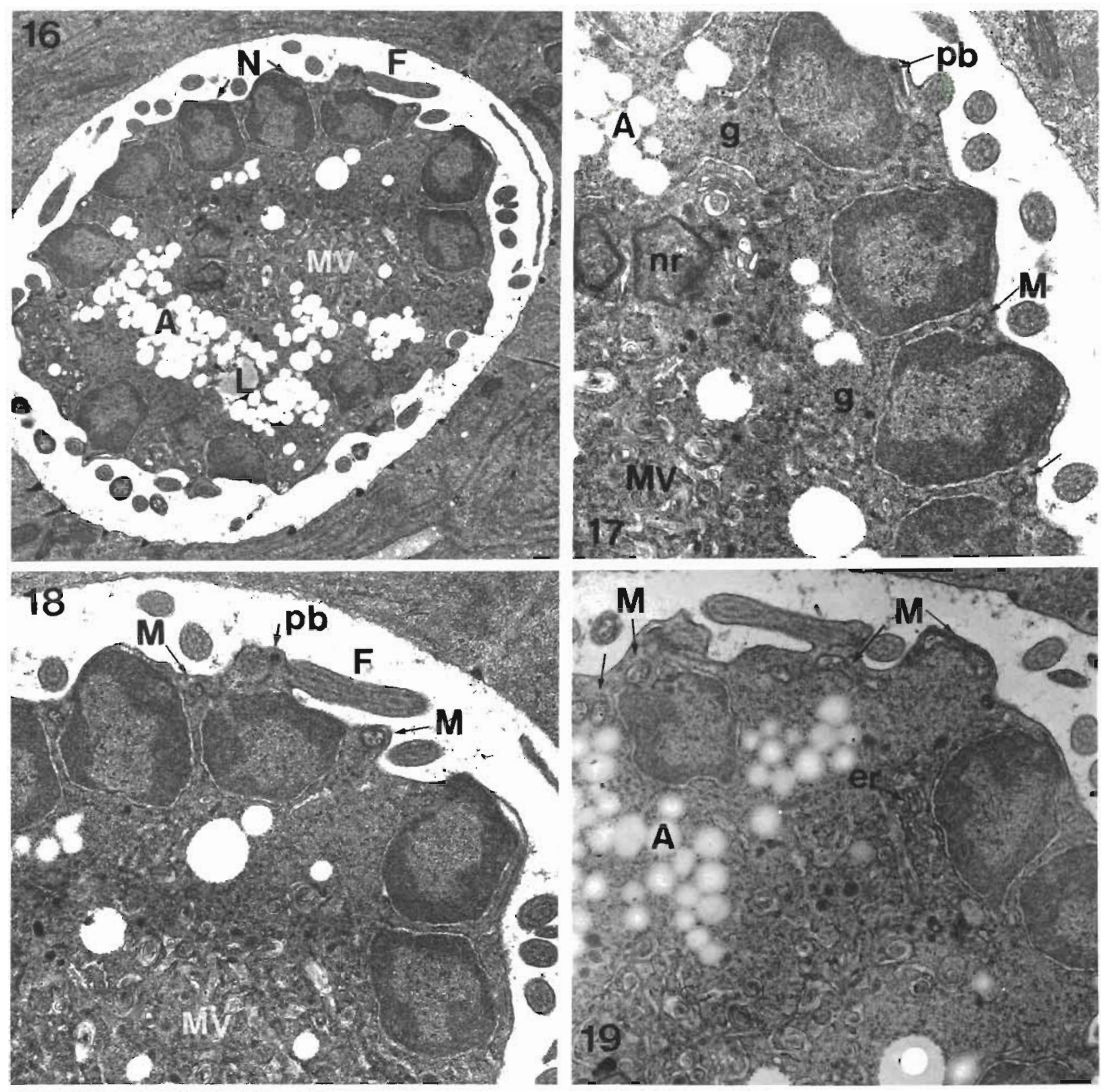

Figs. 16 to 19. Eimeria (s. l.) vanasi. Premature microgamonts. Fig. 16. General view of microgamont with nuclei (N) at the periphery, emerging flagella (F), amylopectin granules (A), lipid vacuole (L) and multimembranous vesicles (MV) (X 6900). Figs. 17, 18 and 19. Enlarged view revealing mitochondria (M), endoplasmic reticulum (er), Golgi apparatus (g), nuclear residues (nr) and basal body of the flagellum $(\mathrm{pb}) .(\times 15500,13800$ and 15000$)$, respectively

gamont, adjacent to microgamete primordia with emergent flagella arising from their basal bodies (Fig. 17). A small mitochondrion accompanies each nucleus (Figs. 17 and 18). Clusters of rounded mitochon- dria were seen at some sites in the peripheral cytoplasm (Fig. 19). Golgi apparatuses and ER tubules occur in the cytoplasm beneath the nuclei (Figs. 17 and 19). The internal cytoplasm contains many amylo-

Figs. 7 to 15. Eimeria (s. 1.) vanasi. Mature macrogamonts (see Fig. 6 for abbreviations). Fig. 7. General view ( $\times 9500$ ). Fig. 8. Macrogamont with scattered storage material granules and with ribosome-lined ducts $(\overline{\times 18} 000)$. Fig. 9. Macrogamont with amylopectin granules aggregated around lipid vacuoles $(\times 10700)$. Fig. 10. Aggregating WF2-like in ER cisternae $(\times 31000)$. Fig. 11. Canaliculli and WF2-like $(\times 31000)$. Figs. 12 to 14 . WF1-like (bold arrow) associated with envelope attachments to the macrogamont's boundary wall and WF2-like (large arrow) $(\times 31000)$. Fig. 15. Details of macrogamont's boundary, envelope and 
pectin granules, a few larger lipid vacuoles, multimembranous vesicles and fragmented residues of nuclei (Figs. 17 and 18).

\section{DISCUSSION}

Eimeria (s. l.) vanasi merozoites developing into macrogamonts are readily distinguishable from those developing into meronts by LM (Landsberg \& Paperna 1987) as well as by TEM (Paperna unpubl.). The latter are attenuated, have a condensed nucleus with heterogeneous chromatin, electron-dense rhoptries and micronemes, and lack lipid vacuoles. Merozoites developing into microgamonts could not be identified either by LM or by TEM.

LM studies of Eimeria vanasi revealed the existence of both epicytoplasmic ('epiepithelial') and intracytoplasmic gamonts (Landsberg \& Paperna 1987). The conspecifity of the epicytoplasmic and intracytoplasmic stages has been discussed elsewhere (Landsberg \& Paperna 1987). Our TEM studies revealed only intracytoplasmic mature gamonts. The PVs containing the young gamonts, and extending beyond the brush border level of the host cell, may have been in the process of emerging to, or submerging from the epicytoplasmic position. If the first interpretation is correct, then the young gamonts represent epicytoplasmic gamonts.

All gamont stages, as well as meronts dividing into gamont-forming merozoites, were enclosed in PV connected to a tubular system extending into the host cell cytoplasm. This system was described and discussed in a previous communication (Paperna \& Landsberg 1987). The tubular system was vestigial or absent in the walls of PVs containing asexual stages (Paperna \& Landsberg 1987, Paperna unpubl.).

Most piscine coccidia lack a hard oocyst wall, and the sporocyst wall assumes the cardinal protective function (Dykova \& Lom 1981). The mature macrogamonts of Eimeria vanasi were enclosed in a superficial envelope, suggesting that they were already macrogametes or zygotes. One or 2 envelopes were seen enclosing macrogametes or zygotes of Goussia iroquoina (Paterson \& Desser 1984) and E. laureleus (Desser \& Li 1984), and 3 in G. zarnowskii (Jastrzebski \& Komorowski 1990). These 'oocystic membranes' were regarded as the fragile oocyst wall of the piscine coccidia. However, the 2 membranes reported to enclose mature $G$. cichlidarum prior to its detachment from the swim bladder epithelium (Paperna et al. 1986) preceded the appearance of a soft but thick oocyst wall (Papema \& Landsberg 1985, Paperna \& Cross 1985). Formation of an envelope around the macrogamete or the zygote also preceded the formation of a hard oocyst wall in saurian coccidia (Paperna 1989, Paperna \& Landsberg 1989).
Oocyst wall membranes in mammalian as well as piscine coccidia were shown to derive from ER associated with the macrogamont plasmalemma (Chobotar et al. 1980, Paterson \& Desser 1981a). In avian and mammalian coccidia WF1 and WF2 (Scholtyseck et al. 1971) contributed material to the formation of hard outer and inner walls (Sibert \& Speer 1980, Chobotar et al. 1980). In $E$. vanasi macrogamonts, the membrane-bounded, ribosome-lined ducts which seem to belong to the rough ER system, and the WF1-like vesicle, appear to open to the point of fusion between the macrogamont plasmalemma and the outer envelope. This may be the point where the latter organelles become involved in membrane formation. There is pronounced variation in the occurrence and fine structure of organelles resembling WF and WF-like organelles among piscine coccidia. Such organelles are unlikely to assume the same function as the WF of higher vertebrate coccidia with hard oocyst walls. Two types of WF, reminiscent of those found in Eimeria vanasi, occur in macrogamonts (reported as sporonts) of Goussia clupearum ('DC' for WF1 and 'WF?' for WF2 in Fig. 7 of Morrison \& Hawkins 1984) and in an undescribed species of epimerian coccidium (from Labrus berggylta, Boulard \& Blanc 1985). In all other species we find either 'dense bodies' ('Mx' and 'MV') of variable size reminiscent of WF1 [in G. iroquoina (Paterson \& Desser 1981a, 1984); in E. laureleus (Desser \& Li 1984), in G. aculeati (Jastrzebski 1989) and in G. zarnowskii (Jastrzebski \& Komorowski 1990)], or a granular or condensed body within the ER cisternae reminiscent of WF2 [in G. cichlidarum (Paperna et al. 1986) and E. sardinae (Morrison \& Hawkins 1984)]. Dense or granular bodies were thought to be involved in sporocyst wall formation in Sarcocystis tenella (Mehlhorn \& Scholtyseck 1974) and Aggregata eberthi (Heller 1969). Material from the 'dense bodies' of $G$. iroquoina and E. laureleus macrogametocytes was re-aggregated in the sporoblasts and sporocysts, but did not seem to participate in sporocyst wall formation (Paterson \& Desser 1981b, Desser \& Li 1984). In G. zamowskil 'dense bodies' located beneath the macrogamonts boundary released their contents into the spaces between the forming envelopes (Jastrzebski \& Komorowski 1990). WF2-like bodies of G. cichlidarum seem to contribute their granular contents to the formation of the sporocyst wall (Paperna \& Landsberg 1985).

Box et al. (1980) commented on the similarities of Sarcocystis $\mathrm{sp}$, and related genera to piscine coccidia, noting the similar development of a fragile, transitory oocyst wall with hard wall formation only at the sporocyst stage, and the lack of a stieda body in the sporocyst. This apparent relationship is only superficial: both types of WF, or at least one (WF1), have been found in macrogamonts of all Sarcocystis sp. studied to date and 
the final, fragile oocyst wall is nevertheless formed by the same or similar process to that observed in Eimeria species, which form a hard wall (Vetterling et al. 1973). Adnuclear bodies were seen before in macrogamonts of Schellackia cf. agamae, their nature and function are unknown (Ostrovska \& Paperna 1987).

The process of microgametogenesis in Eimeria vanasi was similar to that which occurs in the saurian coccidium E. turcicus (Paperna \& Landsberg 1989), in Isospora sp. from passerine birds (Milde 1979) and possibly in the piscine species Calyptospora funduli (as may be evident from Fig. 3 of Hawkins et al. 1983), e.g. the flagellum emerges at the surface of the microgamont before the body of the microgamete, while the microgamont's nucleus does not undergo subdivision prior to microgamete formation. This form of microgametogenesis diverges somewhat from the general coccidian pattern described for many Eimeria spp (Scholtyseck et al. 1972), including piscine spp. (Paterson \& Desser 1981b, Jastrzebski 1989, Jastrzebski \& Komorowski 1990).

Acknowledgement. The study, in part, was supported by the FRG-Israel fund for Agricultural Research in Third World Countries.

\section{LITERATURE CITED}

Boulard, Y., Blanc, I. (1985). Presence d'une coccidie sousepitheliale dans l'intestine de Labrus berggylta. Society of Protozoologists Abstracts, No. 36

Box, E. D., Marchiondo, A. A., Duszynski, D. W., Davis, C. P (1980). Ultrastructure of Sarcocystis sporocysts from passerine birds and opossums: comments on classification of the genus Isospora. J, Parasitol. 66: 68-74

Chobotar, B., Senaud, J., Ernst, J. V., Scholtyseck, E. (1980). Ultrastructure of macrogametes and formation of the aocyst wall of Eimeria papillata in Mus musculus. Protistologica 16: 115-124

Desser, S. S., Li, L. (1984). Ultrastructural observations on the sexual stages and oocyst formation in Eimeria laureleus (Protozoa, Coccidia) of perch, Perca flavescens, from L. Sasajebwum, Ontario. Z. ParasitKde 70: 153-164

Dykova, I., Lom J. (1981). Fish coccidia: critical notes on life cycles, classification and pathogenicity. J. Fish Dis. 4: $487-505$

Hawkins, W E., Solangi, M. A., Overstreet, R. M. (1983). Ultrastructure of the microgamont and microgamete of Eimeria funduli, a coccidium parasitizing killifishes. J. Fish Dis. 6: $45-57$

Heller, G. (1969). Electronenmikroskopische Untersuchungen an Aggregata eberti aus dem Spiraldarm von Sepia officinalis 1. Die Feinstrukturen der Merozoiten, der Makrogameten und der Sporen. Z. ParasitKde 33: 44-64

Jastrzebski, M. (1989). Ultrastructural study on the development of Goussia aculeati, a coccidium parasitizing the three-spined stickleback Gasterosteus aculeatus. Dis. aquat. Org. 6: 45:53

Jastrzebski, M. Komorowski, Z. (1990). Light and electron microscopic studies on Goussia zamowskii (Jastrzebski.
1982): an intestinal coccidium parasitizing the three spined stickleback, Gastrosteus aculeatus (L.). J. Fish Dis. 13 $1-12$

Landsberg, J. H., Paperna, I. (1987). Intestinal infection by Eimeria s. 1. vanasi n. sp. (Eimeridae, Apicomplexa, Protozoa) in cichlid fish. Ann. Parasitol. Hum. Comp. 62: 181-185

Mehlhorn, H., Scholtyseck, E. (1974). Licht- und elektronenmikroskopische Untersuchungen an Entwicklungsstadien von Sarcocystis tennella aus der Darmwand der Hauskatze I. Die Oocysten und Sporocysten. Z. ParasitKde 43: $251-270$

Milde, K. (1979). Light and electron microscope studies on isosporan parasites (Sporozoa) in sparrows (Passer domesticus L.). Protistologica 15: 607-627

Morrison, C. M., Hawkins, W. E. (1984). Coccidians in the liver and the testis of the herring Clupea harengus L. Can. J Zool. 62: 480-493

Ostrovskâ, K., Paperna, I. (1987). Fine structure of gamont stages of Schellackia cf. agamae (Lankesterellidae, Eucoccidia) from the starred lizard Agama stellio. Parasitol. Res. 73: $492-499$

Paperna, I. (1989). Ultrastructure of Eimeria s. 1. sp. infecting the microvillar zone of the intestinal epithelium of geckoes. Ann. Parasitol. Hum. Comp. 84: 89-99

Paperna, I., Cross, R. H. M. (1985). Scanning electron microscopy of gamogony and sporogony stages of Goussia cichlidarum, a coccidian parasite in the swimbladder of cichlid fishes. Protistologica 21: 473-479

Paperna, I., Landsberg, J H. (1985). Ultrastructure of oogony and sporogony in Goussia cichlidarum Landsberg and Paperna, 1985, a coccidian parasite in the swimbladder of cichlid fish. Protistologica 21: 473-479

Paperna, I., Landsberg, J. H. (1987). Tubular formations extending from parasitophorous vacuoles in the gut epithelial cells of cichlid fish infected by Eimeria $(s .1$ ) vanasi. Dis. aquat. Org. 2: 239-242

Paperna, I., Landsberg, J. H. (1989). Fine structure of endogenous stages of Eimeria turcicus developing in the gall bladder epithelium of the gecko Hemidactylus turcicus. Afr. J. Zool. 24: 251-259

Paperna, I., Landsberg, J. H., Feinstein, N. (1986). Ultrastructure of the macrogamont of Goussia cichlidarum Landsberg and Paperna, 1985, a coccidian parasite in the swimbladder of cichlid fish. Ann. Parasitol. Hum. Comp. 61: 351-354

Paterson, W B., Desser, S. S. (1981a). Ultrastructure of macrogametogenesis, macrogametes and young oocysts of Eimeria iroquoina Molnar and Fernando, 1974 in experimentally infected fathead minnows (Pimephales promelas, Cyprinidae). J. Parasitol. 67: 496-504

Paterson, W. B., Desser, S. S. (1981b). An ultrastructural study of microgametogenesis and the microgamete in Eimeria iroquoina Molnar \& Fernando, 1974, in experimentally infected fathead minnows (Pimephalus promelas, Cyprinidael. J. Parasitol. 67: 314-324

Paterson, W. B., Desser, S. S. (1984). Ultrastructural observations on fertilization and sporulation in Goussia iroquoina (Molnar and Fernando, 1974) in experimentally infected fathead minnows (Pimephales promelas, Cyprinidae). J. Parasitol. 70: 703-711

Scholtyseck, E., Mehlhorn, H., Hammond, D. M. (1971). Fine structure of macrogametes and oocysts of coccidia and related organisms. Z. ParasitKde 37. 1-43

Scholtyseck, E., Mehlhorn, H., Hammond, D. M. (1972). Electron microscope studies of microgametogenesis in coccidia and related groups. Z. ParasitKde 38: 95-131 
Sibert, G. J., Speer, C. A. (1980). Fine structure of zygotes and oocysts of Eimeria neischulzi. J. Protozool, 27: 374-379 Veterling. J. M., Pacheco, N. D., Fayer, R. (1973). Fine struc-

Responsible subject Editor: Professor W. Körting, Hannover, Germany ture of gametogony and oocyst formation in Sarcocystis sp. in cell culture. J. Protozool. 20: 613-621

Revised version accepted: August 24, 1990 